\title{
Causes of color
}

\section{Nassau probes why stuff is colored as it is}

The Physics and Chemistry of Color

The Fifteen Causes of Color

by Kurt Nassau

John Wiley \& Sons, New York

Reviewed by Harry J. Leamy

It is no good, I suppose, to write this review in the detached, scholarly tone in which such reviews are properly written. Kurt Nassau has been an AT\&T Bell Labs colleague and pal of mine for some time, and many MRS members know it. Never mind. Kurt has written a very nice book, and it is a pleasure to spread the word.

I first heard about this endeavor during a lunchtime conversation, when my casual query, "What are you up to lately," brought forth the unusual response, "I have just finished a book on color." I thought at first, of course, that he had perhaps written on the topic of elementary particles. But no, it was color-ordinary color-that insignificant sliver of the electromagnetic spectrum that we humans are permitted to perceive, about which he had

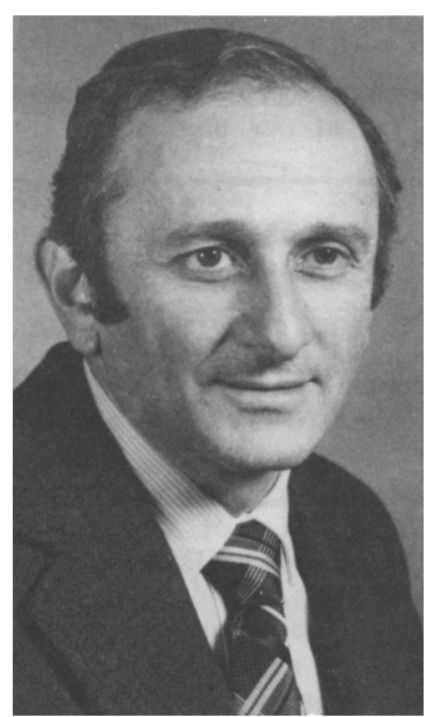

KURT NASSAU written

The color of stuff, and why stuff is colored as it is, must form a large body of literature in chemistry, physics, materials science, and more than a few books on color certainly exist. I have never seen the topic treated as broadly as does Nassau in this volume. First, of course, there is the obligatory discussion of the spectrum, the colors perceived by humans in the visible range, and the chromaticity diagram. Also included in the introductory chapter is a discussion of additive and subtractive color mixing. The introductory chapter is followed by a brief treatment of incandescence, the color of hot stuff, which includes a section on pyrotechnics and reveals the origin of the word limelight as it is used in theatrical reference. Next, Nassau treats the color produced by gas excitations: neon signs, vapor lamps, arcs, lightning, coronas, auroras, and gas lasers. The color produced by transition metals in ligand fields is nicely covered in chapter five, where the behavior of chromium, the impurity responsible for ruby red and emerald green is explained.

Color in organic molecules, including a discussion of organic dyes and dye lasers is treated in chapter 6, while the color of blue sapphire, which is attributable to intervalence charge transfer, is covered in chapter 7. More familiar topics, color in metals and semiconductors are treated in chapter 8. Color centers are covered in chapter 9, which completes that section of the book that might be said to contain material of some familiarity to materials scientists.
Chapters 10,11 , and 12 contain treatments of colors produced by refraction, polarization, scattering, interference, and diffraction. While the physical principles contained in this section will be familiar, the examples that Nassau has selected to illustrate them will delight. Did you know that the color in a bird's feather is usually located in the barbules? The last fourth of the book, save the appendices, is a potpourri of topics: pigments, dyes, biological coloration, color in glass and enamel, photosynthesis, bioluminescence, and the like. Finally, the book contains appendices on units, incandescence equations, atoms and molecules, crystal field theory, band theory, and physical optics, plus a source list for additional reading.

The level of treatment contained in this book is sure to disappoint an expert in any single area that is covered. At the same time, That same expert will, however, delight in the revelations contained in the remainder of the book. The reading is easy; no pencil required. Nassau's style is expository rather than mathematical, and although problems are included at the close of each chapter, I was not compelled to test myself. I was satisfied to have simply read the story. This is not a specialist's book; quite the opposite. It may well be read for the contents of a single chapter, but I'll bet that you can't stop with just one.

\section{BOOK REVIEWS}

\section{Solidification Microstructure: 30 Years after Constitutional Supercooling}

A Special Issue of: Materials Science and Engineering

Editor in Chief: Professor Herbert Herman

Volume 65, No. 1 - July 1984

Guest Editors: Howard Jones and Wilfried Kurz

Elsevier Sequoia S. A. Lausanne

\section{Reviewed by W.A. Bonner}

The present state of understanding of the fundamental aspects of solidification and solidification processes since the derivation of the "constitutional supercooling" criterion is presented in this issue of Materials Science and Engineering." The special issue comprises twenty papers by notable authors. While coverage of such diverse subject matter cannot possibly be comprehensive in the space allotted (200 pages), this volume presents an extremely fine blend of review and original material. Extensive bibliographies acocompany each paper and in this regard the volume is undoubtedly a valuable reference.

Topics presented include a historical overview of crystal growth kinetics and impurity trapping using computer simulation of model systems. Though dendrite formation is an integral part of most solidification discussions, it receives extensive treatment in this volume. Altogether five papers are devoted specifically to dendritic growth. Subjects include dynamics of formation, growth in free, constrained, and undercooled environments, and modeling studies under steady state conditions. Other topics range from microsegregation, eutectic solidification, and heat folw modeling to solidification under microgravity and the microstructure of fusion welds and stir-cast melts. 